\title{
Circadian Clocks and Sleep: Impact of Rhythmic Metabolism and Waste Clearance on the Brain
}

\author{
Urs Albrecht $^{1, *}$ and Jürgen A. Ripperger ${ }^{1}$
}

The rotation of the Earth around its axis causes periodic exposure of half of its surface to sunlight. This daily recurring event has been internalized in most organisms in the form of cellular circadian clock mechanisms. These cellular clocks are synchronized with each other in various ways to establish circadian networks that build the circadian program in tissues and organs, coordinating physiology and behavior in the entire organism. In the mammalian brain, the suprachiasmatic nucleus (SCN) receives light information via the retina and synchronizes its own neuronal clocks to the light signal. Subsequently, the SCN transmits this information to the network of clocks in tissues and organs, thereby synchronizing body physiology and behavior. Disruption of cellular clocks and/or destruction of the synchronization between the clocks, as experienced for instance in jet lag and shift-work conditions, affects normal brain function and can lead to metabolic problems, sleep disturbance, and accelerated neurological decline. In this review, we highlight ways through which the circadian system can coordinate normal brain function, with a focus on metabolism and metabolic astrocyte-neuron communications. Recent developments, for example, on how waste clearance in the brain could be modulated by the circadian clock, will also be discussed. This synthesis provides insights into the impact of metabolism not only on the circadian clock, but also on sleep and how this connection may exacerbate neurological diseases.

The Circadian Clock and Metabolic-Neurophysiological Axis

Epidemiological studies indicate that there are strong interactions between modern lifestyle, sleep deprivation, and circadian misalignment. These reciprocal interactions correlate with various health problems, such as obesity, cancer, addictive behaviors, cardiovascular disease, and neurological disorders (reviewed in [1]). Therefore, a detailed understanding of how environmental conditions such as changes in light exposure, food uptake, and stress affect sleep (see Glossary) and the circadian system is of high priority.

While the mechanisms of how light affects the $\mathbf{S C N}$ are well understood [2], the ones behind the impact the SCN has on sleep, mood, weight regulation, and brain metabolism are only partly clarified. Among the key questions are how metabolism (anabolic and catabolic) is regulated in the brain, and what roles do the neuronal and astrocytic circadian clocks play in the management of energy and waste production in order to orchestrate brain metabolism and function. Metabolism appears to be at the heart of most physiological processes in the peripheral organs and most likely also in the brain. In this Review, we survey the impact of metabolism on the circadian clock, and conversely, ways through which the circadian system can coordinate brain

\section{Highlights}

The physiology and metabolism of organisms are organized in a temporal fashion using cellular circadian clocks for timing. These cellular clocks are synchronized at the tissue level and are in stable phase relationships to other tissues and organs, thereby establishing an organism-wide circadian system.

Temporal organization ensures efficient and highly coordinated metabolism, which is essential for adaptability and robustness of physiological functions under changing environmental conditions. Chronic disturbance of this coordination (e.g., rotating shift work, and chronic jet lag) leads to interference between anabolic and catabolic processes and an increase of metabolic and protein waste products coupled with inefficient clearance. As a consequence, metabolic and neurological diseases may develop.

The glymphatic system has emerged as a waste clearance system for the central nervous system. Its detailed function and temporal coordination for optimal waste clearance is subject of intense research.

${ }^{1}$ Department of Biology, University of Fribourg, Fribourg, Switzerland

\section{${ }^{*}$ Correspondence:}

urs.albrecht@unifr.ch (U. Albrecht). 
metabolism and metabolic astrocyte-neuron communications. We also discuss recent developments in understanding how waste clearance in the brain is related to sleep and the circadian clock. Findings on how the circadian clock modulates the metabolic-neurophysiological axis will hopefully provide novel insights on how sleep, mood, weight regulation, and neurological disorders can be targeted therapeutically, in order to prevent health problems rooted in dysregulation of brain functions.

\section{The Circadian Clock at the Cellular Level}

The molecular mechanism of the circadian clock drives circa 24-h rhythms in a cell-autonomous fashion. It is made up of a transcription-based autoregulatory feedback loop. In mammals, the core clock genes are Bmal1 and Clock (and its homolog Npas2), which activate the expression of Per1, Per2, Cry1, and Cry2. These factors in turn repress their own activation (Figure 1). The nuclear receptors $\mathrm{REV}-\mathrm{ERB} \alpha$ or $\mathrm{ROR} \alpha$ regulate the expression of the activating clock components Bmal1, Clock, and Npas2 by repressing or activating their transcription, respectively, thus completing the core clock mechanism (tilted grey oval in Figure 1) [3]. Additional nuclear receptors, such as PPAR $\alpha$ can activate Bmal1 transcription and this process can be modulated by the PER2 protein (Figure 1). The core clock mechanism can be modulated by molecules (free fatty acids; FFAs) binding to nuclear receptors (e.g., to PPAR $\alpha$ and REV-ERB $\alpha$ ). Additionally, heme and gases (NO and $\mathrm{CO}$ ) can affect the clock components PER, REV-ERB $\alpha$, and NPAS2, which serve as sensors [4-6]. These small molecule-mediated effects can lead to phase shifts of the circadian oscillator similar to the light mediated phase shifts of the clock that are mediated by glutamate [2]. Additionally, metabolites such as lactate and FFAs affect the redox state of the cell and the clock mechanism via $\mathrm{NAD}^{+}$dependent SIRT1 [7,8], modulating transcriptional activity via chromatin remodeling and PER2 deacetylation $[9,10]$. Thus, metabolic changes that affect the redox state have an influence on clock function.

The described clock mechanism can also target so-called clock-controlled genes, which code for proteins important for biological functions including enzymes involved in metabolism (blue oval in Figure 1). Hence, there is a mutual interaction between the circadian clock and metabolism.

Research to identify the genomic targets of the core clock mechanism has revealed that a substantial fraction of genes (5-20\%) in any cell or tissue undergo circadian oscillations at the mRNA level [11], and under diurnal conditions; $82 \%$ of genes are rhythmic in at least one tissue [12]. Furthermore, circadian regulation of gene expression is not limited to transcriptional processes, but includes every regulatory step in gene expression, including splicing, transcriptional termination, polyadenylation, nuclear/cytoplasmic transport, miRNA regulation, translation, protein phosphorylation, and RNA degradation $[3,13]$. Hence, regulation of circadian gene expression includes both transcriptional and post-transcriptional mechanisms, which in turn can be modulated by various factors (including metabolic ones) interacting with these processes.

\section{SCN and Local Brain Clocks}

Cellular clocks form networks and thereby build up circadian programs in tissues, organs, and subsequently, the entire organism. In the brain, various functional nuclei operate either as selfsustaining clocks (SCN), as semiautonomous clocks (OB, DMH, ARC, HB), or as slave oscillators (BNST, AMY, POA, PVN, NAc) [14] (Figure 2A).

The clocks of the nuclei containing semiautonomous or slave clocks are coordinated by the SCN. This coordination ensures optimal function, both physiologically and cognitively, in domains such as appetite, mood, memory, and sleep. In the absence of an efficient SCN

\section{Glossary}

$\beta$-Hydroxybutyrate (BOHB): ketone body generated from FAs during fasting and extended exercise. It functions as an energy source and as signaling molecule that induces the expression of BDNF leading to mitochondrial biogenesis and increased synaptic plasticity. Circadian rhythm: rhythm with a period of about $24 \mathrm{~h}$ that persists under constant conditions (e.g., constant darkness).

Clock controlled genes: genes that are transcriptionally regulated by molecular clock components but are not themselves part of the clock mechanism.

Diurnal or daily rhythm: rhythm with a period of $24 \mathrm{~h}$ that does not persist under constant conditions, but rather requires a daily signal initiating the next cycle of the rhythm. Free-running period: period (cycle length) determined under constant conditions.

Intermittent metabolic switching (IMS): repetitive cycles of a metabolic challenge (fasting and/or exercise) that leads to depletion of liver glycogen stores and elevates circulating ketone bodies, followed by a recovery period (eating, resting and sleeping).

Nuclear receptors: class of proteins that bind various types of molecules including hormones and metabolites. They act as transcriptional activators or repressors.

\section{Paravascular (glymphatic)}

system: waste clearance pathway in the vertebrate central nervous system that depends on glial cells (astrocytes). CSF enters the brain parenchyma via para-arterial influx, which is coupled to a mechanism clearing waste via removal of interstitial fluid and extracellular solutes from the brain and spinal cord. Exchange of solutes is driven by arterial pulsation and temporal (circadian?) expansion and contraction of brain extracellular space. Clearance of waste and soluble proteins is accomplished through convective bulk flow of fluids, facilitated by aquaporin-4 water channels on astrocytes. Phase shift: displacement of a rhythm (e.g., circadian rhythm) to 


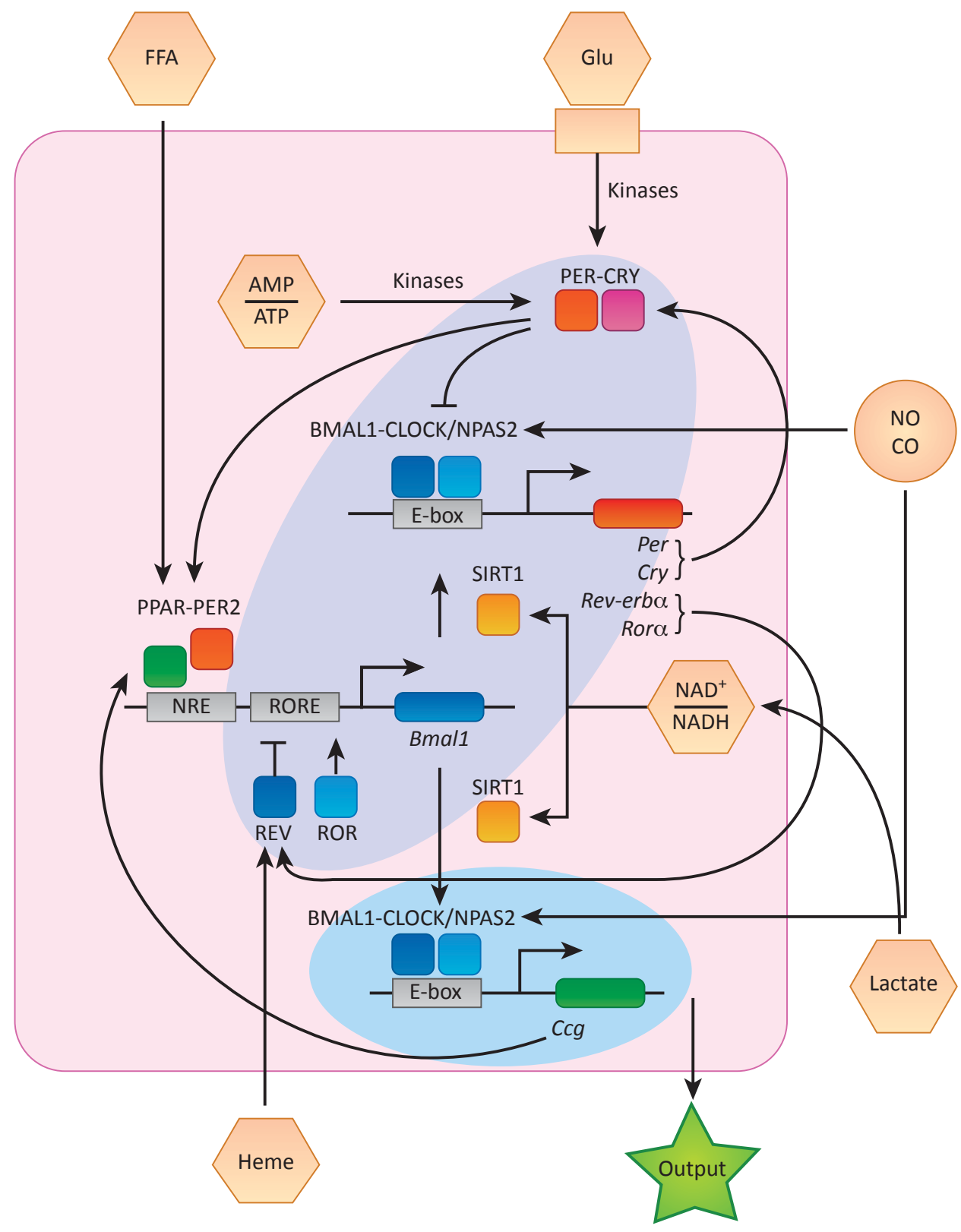

earlier times (advance) or later times

(delay).

Sleep: recurring state characterized

by reduced or absent

consciousness, relatively suspended sensory activity, and inactivity of

nearly all voluntary muscles. It has a circadian component and a

homeostatic component.

Suprachiasmatic nuclei (SCN):

paired nuclei in the ventral part of the hypothalamus lying just above the optic chiasm and on either side of the third ventricle. Each of them comprises $\sim 10000$ neurons in mice and rats. The SCN is the master circadian pacemaker and coordinator of central and peripheral circadian rhythms.

Trends in Neurosciences

Figure 1. The Molecular Circadian Oscillator in a Cell. The diagram depicts a simplified model of the autoregulatory feedback loop of the cellular circadian oscillator (grey shaded tilted oval). Positive factors are depicted in blue, and negative ones in red-brown. The blue horizontal oval depicts the regulation of clock-controlled genes (Ccg; green), which code for a variety of proteins, including transcription factors and enzymes important for the regulation of physiology and metabolism (output of the clock, green star). Various molecules regulate clock proteins directly or indirectly (salmon colored). These molecules may enter the organism from the outside and/or can be products of internal metabolism feeding back to the molecular clock mechanism. Abbreviations: BMAL1, brain and muscle Arnt-like protein-1; CLOCK, circadian locomotor output cycles protein kaput; E-box: binding site for BMAL1 and CLOCK/NPAS2; FFA: free fatty acid; NPAS2, neuronal PAS domain protein 2; NRE: nuclear receptor element; PER, period circadian regulator; PPAR, peroxisome proliferatoractivated receptor; REV, reverse thyroid receptor $\alpha$; ROR, RAR-related orphan receptor; RORE: retinoic orphan receptor binding element; SIRT1, sirtuin 1. 
(A)

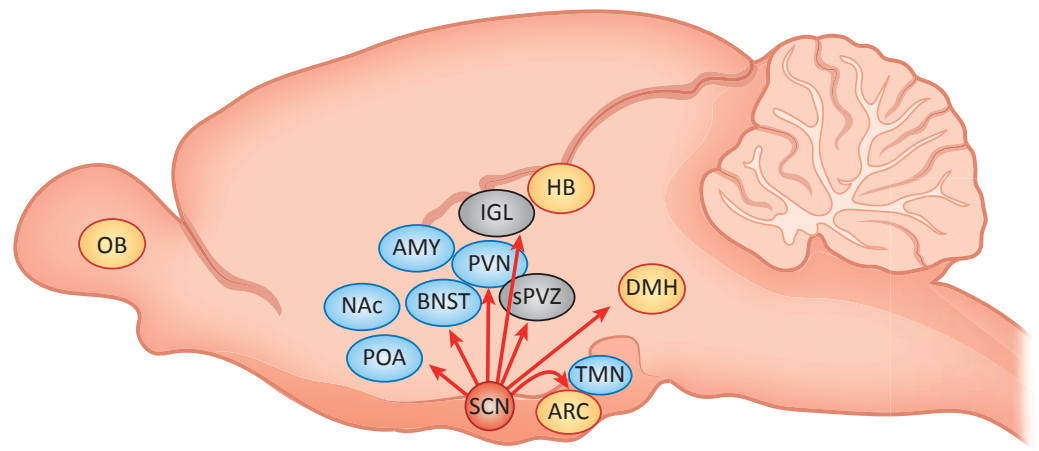

(B)

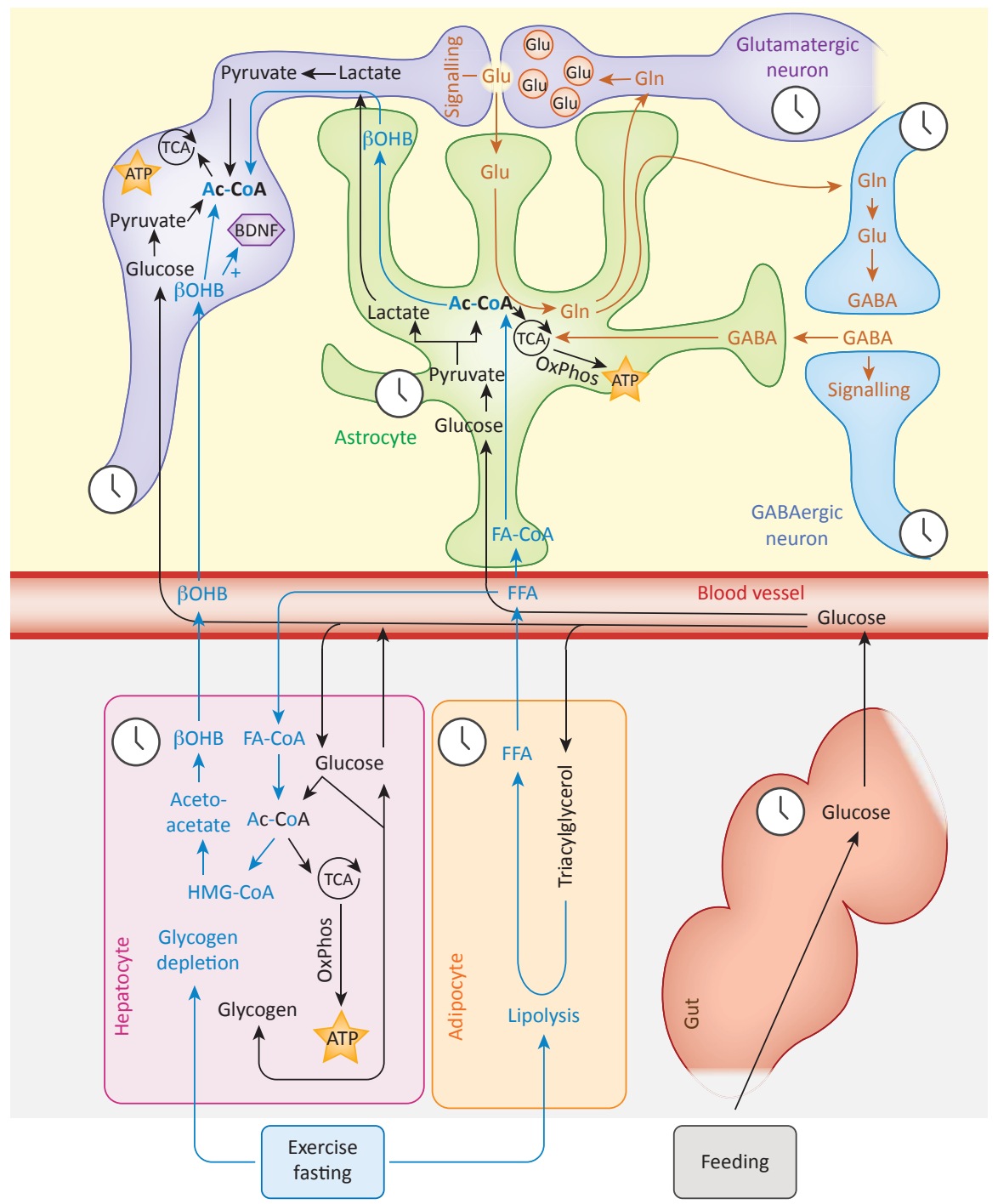

Trends in Neurosciences

Figure 2. Oscillators and Metabolism in the Mammalian Brain. (A) The self-sustaining master clock in the SCN is shown in red, semiautonomous clocks in yellow, and slave oscillators in blue. The different nuclei are important in the (Figure legend continued on the bottom of the next page.) 
signal, regulation of the subordinate brain clocks can be compromised and accordingly affect brain functions. For example, studies in hamsters have shown that induction of arrhythmia by a light pulse compromises hippocampus-dependent memory, but ablation of the SCN in these animals normalizes this effect [15]. This finding indicates that an aberrant SCN signal can have worse outcomes - at least in some cases - than the absence of signaling from the SCN.

Compromising local clock functions, in particular, nuclei can disrupt brain function as well. Deletion of Bmal1 in histaminergic neurons in the tuberomammillary nucleus (TMN) of mice leads to increased histidine decarboxylase expression and higher brain histamine levels during the day [16]. Because histamine enhances wakefulness [17], TMN-Bmal1 knockout mice display more fragmented sleep, prolonged wake periods at night, shallower sleep depth, altered recovery from sleep-deprivation, and impaired memory [16]. Hence, the local TMN clock regulates sleep via histamine levels and modulates the SCN-defined sleep-wake cycle.

Similarly, a recent study indicates that the ARC is more important for the circadian timing system than previously thought. Interruption of SCN-ARC communication does not affect SCN rhythmicity, but causes desynchronization of the ARC, which results in arrhythmicity of activity and physiology [18]. As the ARC is an important metabolic integrator, this finding highlights an intimate interaction between the neural clock system and systemic metabolism. It seems likely that disruption of this SCN-ARC interaction, for instance, by ill-timed eating habits or shift-work conditions, may play a causal role in hypothalamic desynchronization and potentially subsequent disease.

In the future, it will be important to untangle the interdependent network of SCN timing, local clock timing, and sleep/wakefulness. The overarching, general understanding of these interactions at the moment is that the brain is a temporally resonant system, with circadian oscillations at its basis, and upon this basis each brain region is locked to a specific phase, to support and complement other regions. According to this integrative framework, threats to any of the components of the system are expected to disturb normal brain function. This prediction seems to receive support from epidemiological studies [1].

\section{Cooperation of Neurons and Astrocytes in the SCN}

As mentioned above, the SCN are the major synchronizers of circadian rhythms in mammals. This conclusion was reached principally by ablating the SCN region and subsequent reimplantation studies. For example, such an ablation study in hamsters demonstrated that the grafted tissue - but not the remaining host tissue - determined the free-running period [19]. Hence,

regulation of various physiological processes including feeding, mood regulation, and sleep. Red arrows indicate direct connections from the SCN. The circadian clocks of the IGL and SPVZ are contacted by the SCN but their states are not yet characterized (grey). (B) Metabolic interaction between peripheral organs (bottom, grey area) and the brain (top, yellow area) via blood vessels (middle, red area). Feeding triggers the pathways depicted in black, whereas exercise and fasting trigger the pathways depicted in light blue. Both pathways converge at the level of Ac-CoA to keep up ATP production via the TCA cycle. In astrocytes (green) this maintains the Gln-Glu cycle and Gln-Glu-GABA cycle in order to keep up neuronal signaling (orange cyclic pathways in glutamatergic and GABAergic neurons, respectively). In this way, the firing potential of neurons (purple glutamatergic and blue GABAergic) is maintained. The ketone body $\beta O H B$ is used in neurons for ATP production under starvation conditions to maintain the functioning of ionic pumps to keep up ion gradients. Also, $\beta O H B$ positively affects the levels of BDNF, which induces mitochondrial biogenesis and synaptic plasticity. Abbreviations: AcCoA, acetyl-coenzyme A; AMY, amygdala; ARC, arcuate nucleus; BDNF, brain-derived neurotrophic factor; BNST, bed nucleus of the stria terminalis; $\mathrm{DMH}$, dorsomedial hypothalamus; FFA, free fatty acid; GABA, $\gamma$-aminobutyric acid; HB, habenula; HMG-CoA, 3-hydroxy-3-methyl-glutaryl-coenzyme A; IGL, intergeniculate leaflet; NAc, nucleus accumbens; $\mathrm{OB}$, olfactory bulb; $\beta \mathrm{OHB}, \beta$-hydroxybutyrate; OxPhos, oxidative phosphorylation; POA, preoptic area; PVN, paraventricular nucleus; SCN, suprachiasmatic nucleus; sPVZ, periventricular zone; TCA, tricaboxylic acid; TMN, tuberomammillary nucleus. Adapted, with permission, from [31,53,54]. 
the signals from the SCN seem dominant over the rest of the body. Even a fetal SCN-derived neuronal cell line rescued the ablated SCN region in rats [20]. Nevertheless, this rescue was less efficient compared to intact or dispersed fetal SCN tissue [21]. Could, therefore, other cell types apart from neurons contribute to circadian rhythm generation? Immunohistochemical studies revealed that the SCN region is denser in glial fibrillary acidic protein (GFAP)-like-positive cells compared to the surrounding tissues [22]. This observation suggested (but did not prove) involvement of GFAP-positive astrocytes in circadian rhythm generation. Plausible functions of astrocytes would involve the metabolic coupling to neurons and the clearance of neurotransmitters (see below).

Monitoring of $\left[\mathrm{Ca}^{2+}\right]$ in astrocytes and in $\gamma$-aminobutyric acid (GABA) secreting neurons of the dorsal SCN revealed that these cell types had roughly opposite phases of activity [23]. In addition, glutamate was released by the astrocytes and taken up rhythmically by the neurons via time-of-day dependent activity of the excitatory amino acid transporter (EAAT)3. Interference with either the circadian activity of astrocytes in the dorsal SCN, or the flux of glutamate between the different cell types interfered with the proper functioning of the circadian timing system. As a possible interpretation, it was proposed that during the circadian night, glutamate is not taken up by the neurons but $\mathrm{Ca}^{2+}$ is, provoking a hyperpolarization of the GABAergic SCN neurons [23]. In contrast, during the circadian day, the conditions reverse, leading to a depolarization of the same neurons and consequently increased neuronal firing rates.

What is the potential advantage of using astrocytes to synchronize neurons? In theory, inhibition of neuronal activity could result from the secretion of GABA by GABAergic SCN neurons (Figure 2B, right, orange pathway). However, this would be dependent on the individual neuronal activity and consequently would probably be too unreliable. Hence, astrocytes may be necessary to synchronize multiple neurons without interfering with their overall activity and consequently to fine tune the system. Astrocytes in the SCN have been described as a connected network communicating via connexin 43 gap junctions [24]. This tight coupling could coordinate the activity of the entire astrocyte network. Similarly, spikes of glutamate released by astrocytes in the cortex have been suggested to synchronize the activity of neurons and generate slow wave sleep $(\sim 1 \mathrm{~Hz})$ oscillations [25]. Taken together, astrocytes and neurons are rhythmically interconnected, and their coupling affects the polarization state of SCN neurons in order to optimize circadian rhythms in the dorsal part of the SCN [23] (Figure 2B, orange pathways).

Two additional studies analyzed the impact of arrhythmic astrocytes in the SCN on the circadian timing system of mice $[26,27]$. Both studies found a lengthening of the free-running period upon specific deletion of Bmal1 in astrocytes of the SCN. Hence, the rhythmicity of the astrocytes is not a prerequisite for the functioning of the circadian timing system, but rather necessary to adjust the period length. An additional function of astrocytes is to absorb neurotransmitters such as GABA released from the neurons into the synaptic cleft. This uptake was impaired in Bmal1-deficient astrocytes, and higher levels of GABA were measured in the SCN of astrocyte-specific knockout mice [26]. Finally, the application of several GABA receptor antagonists could reverse the period lengthening of the free-running locomotor activity of these mice [26]. Hence, astrocytes can fine-tune the period of SCN neurons by neurotransmitter reuptake.

Taken together, these results, from three independent studies, confirm that astrocytes have circadian rhythms that are an integral part of the circadian timing system in the SCN and therefore affect the synchronization of rhythms across the entire animal. As such, the rhythmic 
activity of astrocytes in the SCN could have also an impact on the circadian component of the sleep-wake cycle $[23,28]$.

\begin{abstract}
Metabolism in the Brain: Modulation by Daily Activity and Feeding, and Dependence on Peripheral Organs

It is generally accepted that the circadian clock enables organisms to predict daily recurring events. This view is supported, for example, by a recent observation, that clock-driven vasopressin (VP) neurons mediate anticipatory thirst in mice just before sleep. Notably, thirst was not motivated by physiological need, but rather was dependent on the SCN projections to the organum vasculosum lamina terminalis (OVLT), where VP activates nonselective cation channels via $\mathrm{V} 1$ a receptors to induce water intake. This SCN clock regulating thirst anticipation is probably important in order to protect against overnight dehydration [29].
\end{abstract}

In contrast to anticipatory thirst, food anticipation is independent of a functional SCN [30]. Food anticipation is observed under restricted food availability (often coupled with caloric restriction) when food is available only at a specific time. This way of feeding can also be viewed as intermittent fasting, which is known to have profound effects on neuroplasticity and brain health (reviewed in [31]). Time-restricted feeding every day during the early light phase with caloric restriction (30\% less than ad libitum consumption) is inducing intermittent metabolic switching (IMS). The fasting and/or exercise period depletes liver glycogen stores and elevates circulating ketone body levels. The following recovery period (eating, resting, and sleeping) restores glycogen in the liver and reduces ketone body levels in the circulation. Hence, there is a switch between glucose and ketone body usage. Ketone body levels, especially of $\boldsymbol{\beta}$-hydroxybutyrate $(\beta \mathrm{OHB})$, rise before feeding time in rats and mice under these conditions (reviewed in [31]).

Mice that lack the clock gene Per2 in the liver did not display a rise in $\beta O H B$ levels before food availability and showed no food anticipatory activity. The phenotype, however, was rescued by perfusing $\beta \mathrm{OHB}$ into the blood prior to food availability [32]. This observation indicated that $\beta \mathrm{OHB}$ is important for food anticipation and that IMS and the clock are linked directly or indirectly via the Per2 gene. This finding also correlated with the observation that the ratelimiting enzymes for ketone body synthesis were altered in their expression in Per2 liver knockout mice [32]. $\beta \mathrm{OHB}$ may serve as a daily signal in order to induce food-seeking behavior before glucose levels can no longer be maintained at a constant level. Hence, peripheral tissues such as the liver influence the brain, via metabolic signaling, to induce specific, time-regulated behaviors.

The fuel supply of the brain is entirely dependent on peripheral tissues, mostly the liver and adipose tissues (Figure 2B). To sustain neuronal signaling, the glutamine-glutamate cycle between astrocytes and neurons (orange pathway in Figure 2B) needs a constant energy supply. This homeostasis is achieved through coupling this process to usage of glucose and FAs via the tricarboxylic acid (TCA) cycle (black and blue pathways in Figure 2B). Under feeding conditions, glucose is used (black pathways in Figure 2B), ensuring ATP production. Under fasting and/or exercise conditions (blue pathways in Figure 2B), lipolysis leads to release of FFAs from adipose tissue into the circulation, from where they are taken up by astrocytes and used for ATP generation and $\beta \mathrm{OHB}$ production to run the glutamine-glutamate cycle, and/or provide energy to neurons. Hepatocytes (the main cells of the liver, and which have high metabolic activity) take up FFAs as well and supply neurons with energy in form of $\beta \mathrm{OHB}$ (blue pathways in Figure 2B). Of note, $\beta \mathrm{OHB}$ appears to have a positive influence on brain-derived neurotrophic factor (BDNF) production, correlating with the positive effects of IMS on 
neuroplasticity (reviewed in [31]). Hence, daily cycles in the levels of metabolites in the blood affect neuronal metabolism and function.

\section{Timed Clearance of Metabolic Waste from the Brain}

The functionality of the brain depends on the delivery of nutrients, salts, and vitamins by peripheral organs. Nutrients are transported via the vascular system that branches into capillaries to penetrate the brain. The blood itself, however, is not in direct contact with brain cells. It is separated from the brain parenchyma by two distinct barriers: the blood-brain barrier (BBB) and the blood-cerebrospinal fluid barrier (B-CSF). These barriers are critical in order to maintain the ionic and biochemical composition of the four fluid compartments of the brain: intracellular fluid (ICF) (60-68\%), interstitial fluid (ISF) (extracellular fluid, 12-20\%), blood (10\%), and CSF (10\%) (Figure 3A). To reach the brain and nourish brain cells such as neurons and astrocytes, nutrients have to pass the BBB and the B-CSF. This transport is accomplished via active transport systems involving various receptors and transporters.

Likewise, clearance of metabolic waste from the brain is essential in order to keep the biochemical milieu of the brain capable of controlling important physiological actions. Daily fluctuations in the relative levels of metabolites, leptin, and cytokines between the blood and the CSF have been observed [33,34], suggesting that there is a daily rhythm in the permeability of the B-CSF barrier. This claim is supported by the observation that the choroid plexus, an important component of the B-CSF barrier, contains circadian clock activity [35], potentially regulating timed fluid exchange between the blood and CSF. A recent study using the fruit fly as a model system revealed that a circadian clock in glial cells of the hemolymph-brain barrier regulated xenobiotic efflux [36]. Therefore, it is tempting to speculate that in mammals, similar to fruit flies, glial cells including astrocytes may regulate BBB function, thereby modulating metabolic waste clearance (Figure 3B).

Peripheral organs clear their waste via the lymphatic system. The brain is a highly active metabolic tissue and does not have direct access to the lymphatic system. Given that, one wonders how the brain removes its waste products. First indications for possible wasteclearance routes have come from experiments in which large proteins were injected into the CSF. These proteins were identified after some time in the perivascular space, which surrounds the cerebral blood vessels $[37,38]$. This finding suggests that fluid circulation through the central nervous system (CNS) may occur via paravascular pathways, surrounding the vasculature in the brain. More recent studies have characterized specific systems that remove waste products such as glucose, lactate, and $\beta$-amyloid from the brain $[39,40]$. It appeared that specialized astrocytes have a central function in this context, serving as filters that control the flux of compounds smaller than $100 \mathrm{kDa}$ via their aquaporin (AQP)-4 channels (reviewed in $[41,42])$ (Figure 3B). The vascular endothelial cells bordering the paravascular space do not have AQP4 channels and, hence, fluid from the blood stream cannot move directly into the brain. Therefore, liquid has to flow through the paravascular space and then through the astrocytes to gain access to the brain tissue (Figure 3B).

In studies aimed to examine product clearance via paravascular pathways, pumping of blood through the arteries appeared to propel large quantities of CSF into the paravascular space. The CSF then moved via astrocytes through the brain tissue, picking up discarded proteins such as $\beta$-amyloid [43,44]. The volume of the paravascular system (also termed glymphatic system), and with it the flow rate of the CSF appeared to vary according to time of day. These daily variations were correlated with the sleep state. During sleep, the volume of the interstitial space increased substantially ( $60 \%$, according to the study) with concomitantly faster 


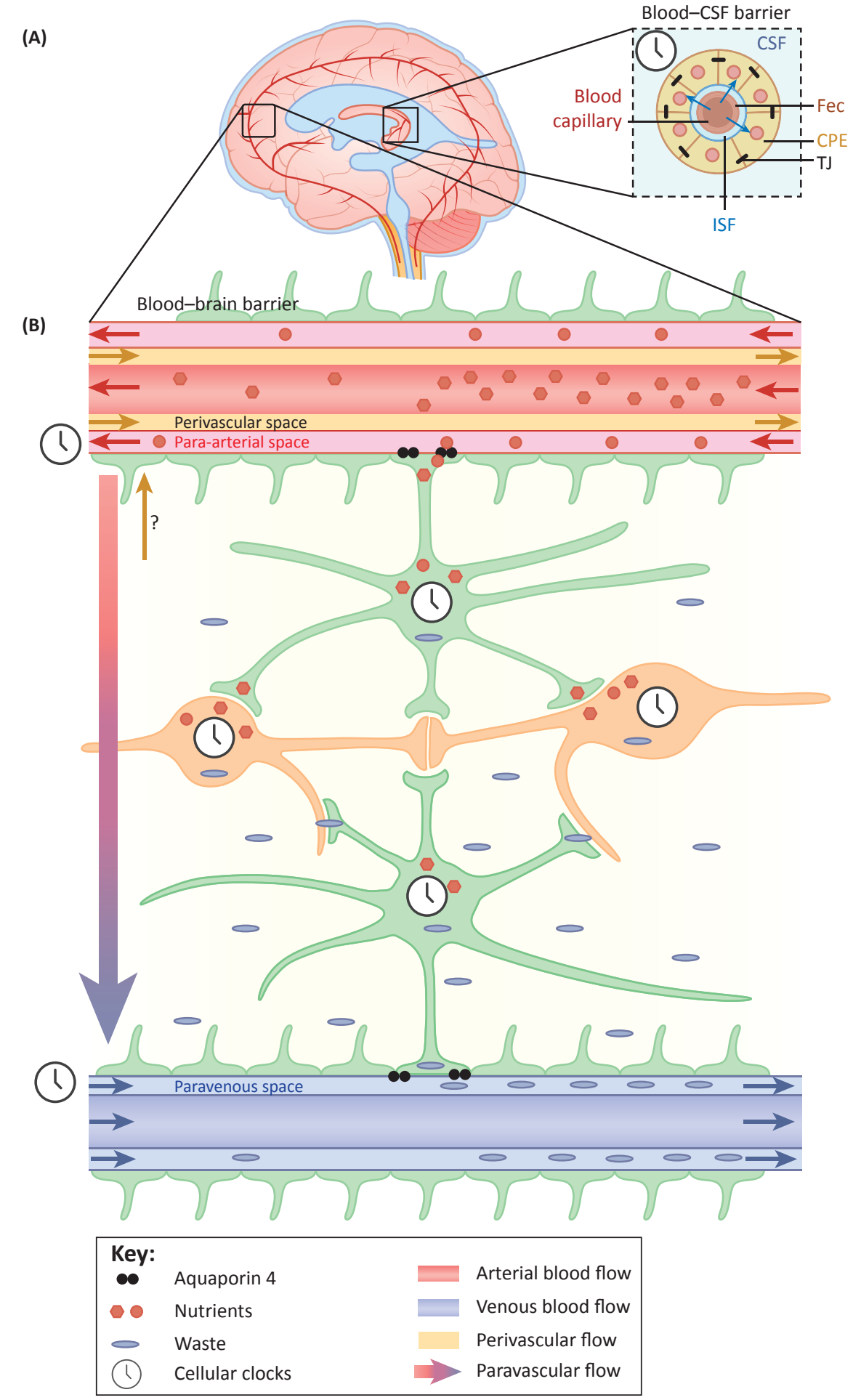

Trends in Neurosciences

Figure 3. Schematic of the Paravascular (Glymphatic) Waste Clearance System with Potential Circadian Clocks. (A) Representation of the fluid compartments and barriers of the brain. (B) Waste clearance route through the (Figure legend continued on the bottom of the next page.) 
clearance of waste products such as $\beta$-amyloid [45]. These changes appeared to be controlled by noradrenergic signaling. It is not known, however, whether and how such signaling affects astrocytes or their AQP4 channels to allow an influx of water into the interstitial space, and whether this process might be under control of the circadian clock.

\section{Diurnal Variations of Liquid Flow in the Brain}

Fluids entering the brain via the para-arterial space, flushing through the brain and collecting waste, leave the brain tissue via the paravenous space (Figure 3B) [41,42]. Eventually, the combined paravascular waste liquids reach the neck region, where they enter the lymph system to flow back into the general blood circulation (reviewed in [41]). Alternatively, a perivascular pathway, located within the arterial tunica media (Figure 3B), has been described with a solute flux along arteries in direction opposite to that of the blood $[46,47]$. Whether this pathway could clear waste products efficiently is under debate (reviewed in [41]). One possibility is that the paravascular and the perivascular systems are two independent but somehow intermingled transport systems. It is also possible that there is a rhythmic component involved in the selection of the direction of waste disposal. The flow rates and direction of the cilia-based flow of CSF in the third ventricle displayed a diurnal change [48], suggesting time-of-day dependence upon CSF flow. Recently, the cochlea has been shown to be under circadian control affecting its sensitivity to noise trauma. Since the inner ear fluids of the cochlea are in continuity with the CSF, there could be similar mechanisms controlling cochlear fluids $[49,50]$. However, further research is necessary to understand the regulation of flow directions and hence clearance of CSF and waste from the brain.

As mentioned, the glymphatic system seems to be regulated by sleep state, raising the interesting hypothesis that a major function of sleep would be to clear out metabolic waste products from the brain through this system $[38,45]$. Similar to the processes found at the circuit level in the SCN and neocortex, it is tempting to speculate that astrocytes - rather than neurons - are the main sensors and coordinators of waste disposal. By the same token, a change in the concentration of waste products in the ISF could represent a signal indicating sleep need. Based on several observations showing that metabolism and sleep may be related, it is postulated that restoration of brain energy metabolism may be one of the functions of sleep [51]. Clearance of lactate by the glymphatic system correlates well with the sleep phase [40], and this clearance could be governed by local astrocytes according to their circadian state. It will be interesting to challenge this concept in the future.

\section{Concluding Remarks and Future Perspectives}

The mammalian circadian system is often seen as a network of circadian clocks that are governed by the SCN. Recent developments, however, indicate that peripheral clocks strongly influence the circadian system, mainly through metabolism, which is in a mutual regulatory feedback loop with the cellular circadian clocks (Figure 1). Since the brain is entirely dependent on energy supplied by peripheral organs, proper functioning of the brain depends on these organs and the daily energy supplied by them. Hence, effective communication mechanisms between the brain and the periphery, for instance, via hormonal and small-molecule signals

blood-brain barrier with a paravascular flow (red-blue shaded arrow) from arteries (red) to veins (purple). Astrocytes (green) take up fluid from the para-arterial space via aquaporin 4 channels and distribute it to neurons (orange) and the intercellular space. Waste loaded fluid is then cleared by astrocytes via aquaporin 4 into the paravenous space and transported out of the brain. A perivascular flow (yellow arrows) is also hypothesized. Abbreviations: CPE, choroid plexus epithelial cell; CSF, cerebrospinal fluid; Fec, fenestrated endothelial cell; ISF, interstitial fluid; TJ, tight junction. Adapted, with permission, from $[41,55]$. 
(e.g., metabolites), to ensure a steady flow equilibrium of nutrients and of waste clearance, are crucial for optimal brain function and ultimately for survival. The brain has a privileged position of regulating uptake of molecules from the bloodstream through specific mechanisms in the BBB and the B-CSF. Recent evidence has emerged $[35,36]$ showing that circadian clocks exist at these barriers between the periphery and the brain. These clocks may gate in a temporally controlled manner by which molecules can enter and/or exit the brain, and possibly also synchronize the entry/exit of subsets of these molecules. This regulation appears to be essential for normal brain function. Overloading the brain with nutrients when they are not needed, for instance, could lead to excess waste production and accumulation of toxic substances, such as reactive oxygen species. These can modify proteins and may impair brain function and even destroy neurons. The impact of such toxic accumulation may be first noticed as memory loss and sleep disturbance. In this framework, sleep can be seen (at least in part) as a consequence of metabolic regulation, aimed to protect cells in the brain [52].

Overall, brain fluid management is emerging as a key factor in brain function [41]. Our understanding of the systems mediating liquid flux through the brain, for instance, the paravascular (glymphatic) system, is still evolving, and future studies will help clarify the driving forces behind brain fluid influx and outflow. The circadian clock likely plays an important role in this context, which relates to the more general purpose of the clock of managing and coordinating daily cycles in most, if not all physiological and biochemical processes across the organism. Another goal for future experiments is to further elucidate transport mechanisms acting at the BBB and B-CSF, including whether and how these are regulated by the circadian clock (see Outstanding Questions).

Altogether, the circadian clock regulates a variety of crucial processes in the brain, including sleep, brain metabolism, and maintenance of flow balance of compounds into and out of the brain. Disruption of the circadian clock, and/or any of the signaling pathways regulated by it, can lead to inefficient anabolic and catabolic biochemical processes. These metabolic impairments and other clock-related dysfunctions are increasingly recognized as key players in the etiology of sleep disturbances, mood alternations, and a variety of neuropsychiatric and neurological diseases.

\section{Acknowledgments}

We thank Cressida Harvey for critically reading the manuscript. The work was supported by the Swiss National Science Foundation (31003A_166682), the Velux Foundation (995), and the University of Fribourg.

\section{References}

1. Roenneberg, T. and Merrow, M. (2016) The circadian clock and human health. Curr. Biol. 26, R432-443

2. Hughes, S. et al. (2015) Photic regulation of clock systems. Methods Enzymol. 552, 125-143

3. Takahashi, J.S. (2017) Transcriptional architecture of the mammalian circadian clock. Nat. Rev. Genet. 18, 164-179

4. Dioum, E.M. et al. (2002) NPAS2: a gas-responsive transcription factor. Science 298, 2385-2387

5. Kaasik, K. and Lee, C.C. (2004) Reciprocal regulation of haem biosynthesis and the circadian clock in mammals. Nature 430, 467-47-

6. Yin, L. et al. (2007) Rev-erbalpha, a heme sensor that coordinates metabolic and circadian pathways. Science 318, 1786-1789

7. Nakahata, Y. et al. (2009) Circadian control of the NAD+ salvage pathway by CLOCK-SIRT1. Science 324, 654-657

8. Ramsey, K.M. et al. (2009) Circadian clock feedback cycle through NAMPT-mediated NAD+ biosynthesis. Science 324, 651-654
Outstanding Questions

How do the clocks of the various brain nuclei communicate and synchronize with each other?

How do peripheral clocks and their metabolites regulate clocks in brain nuclei?

How are astrocytes synchronized to affect circadian rhythms?

What are the mechanisms regulating the diurnal changes in ISF volume?

How do neuromodulatory signals, for instance noradrenergic tone, modulate astrocytes and thereby regulate brain liquid flow?

How is the flow direction of CSF regulated?

Are there local tissue clocks in the glymphatic (paravascular) system itself, and if so, how exactly do they regulate brain liquid flow and brain waste clearance? 
present throughout the mammalian brain? Eur. J. Neurosci. 25, 3195-3216

15. Fernandez, F. et al. (2014) Circadian rhythm. Dysrhythmia in the suprachiasmatic nucleus inhibits memory processing. Science 346, 854-857

16. Yu, X. et al. (2014) Circadian factor BMAL1 in histaminergic neurons regulates sleep architecture. Curr. Biol. 24, 2838-2844

17. Parmentier, R. et al. (2002) Anatomical, physiological, and pharmacological characteristics of histidine decarboxylase knock-out mice: evidence for the role of brain histamine in behavioral and sleep-wake control. J. Neurosci. 22, 7695-7711

18. Buijs, F.N. et al. (2017) Suprachiasmatic nucleus interaction with the arcuate nucleus; essential for organizing physiological rhythms. eNeuro Published online March 24, 2017. http://dx. doi.org/10.1523/ENEURO.0028-17.2017

19. Ralph, M.R. et al. (1990) Transplanted suprachiasmatic nucleus determines circadian period. Science 247, 975-978

20. Earnest, D.J. et al. (1999) Immortal time: circadian clock properties of rat suprachiasmatic cell lines. Science 283,693-695

21. Silver, R. et al. (1990) Dispersed cell suspensions of fetal SCN restore circadian rhythmicity in SCN-lesioned adult hamsters. Brain Res. 525, 45-58

22. Morin, L.P. et al. (1989) Two brain nuclei controlling circadian rhythms are identified by GFAP immunoreactivity in hamsters and rats. Neurosci. Lett. 99, 55-60

23. Brancaccio, M. et al. (2017) Astrocytes control circadian timekeeping in the suprachiasmatic nucleus via glutamatergic signaling. Neuron 93, 1420-1435

24. Welsh, D.K. and Reppert, S.M. (1996) Gap junctions couple astrocytes but not neurons in dissociated cultures of rat suprachiasmatic nucleus. Brain Res. 706, 30-36

25. Poskanzer, K.E. and Yuste, R. (2016) Astrocytes regulate cortica state switching in vivo. Proc. Natl. Acad. Sci. U. S. A. 113, E26752684

26. Barca-Mayo, O. et al. (2017) Astrocyte deletion of Bmal1 alters daily locomotor activity and cognitive functions via GABA signalling. Nat. Commun. 8, 14336

27. Tso, C.F. et al. (2017) Astrocytes regulate daily rhythms in the suprachiasmatic nucleus and behavior. Curr. Biol. 27, 1055-106

28. Borbely, A.A. et al. (2016) The two-process model of sleep regulation: a reappraisal. J. Sleep Res. 25, 131-143

29. Gizowski, C. et al. (2016) Clock-driven vasopressin neurotrans mission mediates anticipatory thirst prior to sleep. Nature 537 685-688

30. Stephan, F.K. et al. (1979) Anticipation of 24-hr feeding schedules in rats with lesions of the suprachiasmatic nucleus. Behav. Neura Biol. 25, 346-363

31. Mattson, M.P. et al. (2018) Intermittent metabolic switching, neuroplasticity and brain health. Nat. Rev. Neurosci. 19, 63-80

32. Chavan, R. et al. (2016) Liver-derived ketone bodies are necessary for food anticipation. Nat. Commun. 7, 10580

33. Pan, W. and Kastin, A.J. (2001) Diurnal variation of leptin entry rom blood to brain involving partial saturation of the transport system. Life Sci. 68, 2705-2714

34. Pan, W. et al. (2002) Selected contribution: circadian rhythm of tumor necrosis factor-alpha uptake into mouse spinal cord. $J$. Appl. Physiol. 92, 1357-1362

35. Myung, J. et al. (2018) The choroid plexus is an important circadian clock component. Nat. Commun. 9, 1062
36. Zhang, S.L et al (2018) A circadian clock in the blood-brain barrier regulates xenobiotic efflux. Cell 173, 130-139

37. Foldi, M. et al. (1968) Lymphatic drainage of the brain. Experientia 24, 1283-1287

38. Rennels, M.L. et al. (1985) Evidence for a 'paravascular' fluid circulation in the mammalian central nervous system, provided by the rapid distribution of tracer protein throughout the brain from the subarachnoid space. Brain Res. 326, 47-63

39. Ball, K.K. et al. (2010) Trafficking of glucose, lactate, and amyloidbeta from the inferior colliculus through perivascular routes. J. Cereb. Blood Flow Metab. 30, 162-176

40. Lundgaard, I. et al. (2017) Glymphatic clearance controls statedependent changes in brain lactate concentration. J. Cereb. Blood Flow Metab. 37, 2112-2124

41. Bacyinski, A. et al. (2017) The paravascular pathway for brain waste clearance: current understanding, significance and controversy. Front. Neuroanat. 11, 101

42. Plog, B.A. and Nedergaard, M. (2017) The glymphatic system in central nervous system health and disease: past, present, and future. Annu. Rev. Pathol. 13, 379-394

43. lliff, J.J. et al. (2012) A paravascular pathway facilitates CSF flow through the brain parenchyma and the clearance of interstitial solutes, including amyloid beta. Sci. Transl. Med. 4, 147ra111

44. Deane, R. et al. (2008) apoE isoform-specific disruption of amyoid beta peptide clearance from mouse brain. J. Clin. Invest. 118, $4002-4013$

45. Xie, L. et al. (2013) Sleep drives metabolite clearance from the adult brain. Science 342, 373-377

46. Carare, R.O. et al. (2008) Solutes, but not cells, drain from the brain parenchyma along basement membranes of capillaries and arteries: significance for cerebral amyloid angiopathy and neuroimmunology. Neuropathol. Appl. Neurobiol. 34, 131-144

47. Weller, R.O. et al. (2009) Lymphatic drainage of the brain and the pathophysiology of neurological disease. Acta Neuropathol. 117, $1-14$

48. Faubel, R. et al. (2016) Cilia-based flow network in the brain ventricles. Science 353, 176-178

49. Meltser, I. et al. (2014) TrkB-mediated protection against circadian sensitivity to noise trauma in the murine cochlea. Curr. Biol. 24, 658-663

50. Park, J.S. et al. (2016) Identification of a circadian clock in the inferior colliculus and its dysregulation by noise exposure. J. Neurosci. 36, 5509-5519

51. Benington, J.H. and Heller, H.C. (1995) Restoration of brain energy metabolism as the function of sleep. Prog. Neurobiol. $45,347-360$

52. Albrecht, U. (2012) Circadian rhythms and sleep - the metabolic connection. Pflugers Arch. 463, 23-30

53. Dibner, C. et al. (2010) The mammalian circadian timing system: organization and coordination of central and peripheral clocks. Annu. Rev. Physiol. 72, 517-549

54. Walls, A.B. et al. (2015) The glutamine-glutamate/GABA cycle: function, regional differences in glutamate and GABA production and effects on interference with GABA metabolism. Neurochem. Res. 40, 402-409

55. Jessen, N.A. et al. (2015) The glymphatic system: a beginner's guide. Neurochem Res, 40, 2583-2599 